\title{
Pedigree analysis of Mexican families with Fabry disease as a powerful tool for identification of heterozygous females
}

\author{
B.E. Gutiérrez-Amavizca ${ }^{1,2}$, R. Orozco-Castellanos ${ }^{3,4}$, \\ J. R. Padilla-Gutiérrez ${ }^{5}$, Y. Valle ${ }^{5}$ and L.E. Figuera ${ }^{1,2}$ \\ ${ }^{1}$ División de Genética, Centro de Investigación Biomédica de Occidente, \\ Instituto Mexicano del Seguro Social, Guadalajara, Jalisco, México \\ ${ }^{2}$ Doctorado en Genética Humana, Centro Universitario de Ciencias de la Salud, \\ Universidad de Guadalajara, Guadalajara, Jalisco, México \\ ${ }^{3}$ Centro del Riñón, Guadalajara, Jalisco, México \\ ${ }^{4}$ División de Nefrología y Trasplantes, Unidad Médica de Alta Especialidad, \\ Centro Médico Nacional de Occidente, Instituto Mexicano del Seguro Social, \\ Guadalajara, Jalisco, México \\ ${ }^{5}$ Departamento de Clínicas Médicas, \\ Centro Universitario de Ciencias de la Salud, Universidad de Guadalajara, \\ Guadalajara, Jalisco, México
}

Corresponding author: L.E. Figuera

E-mail: luisfiguera@yahoo.com

Genet. Mol. Res. 13 (3): 6752-6758 (2014)

Received May 28, 2013

Accepted September 25, 2013

Published August 28, 2014

DOI http://dx.doi.org/10.4238/2014.August.28.19

\begin{abstract}
Fabry disease (FD) is an X-linked lysosomal storage disease caused by $\alpha$-galactosidase A deficiency; in contrast to other $\mathrm{X}$-linked diseases, heterozygous females can be as affected as men. The construction and analysis of a family pedigree is a powerful tool to aid clinicians in diagnosis, establishment of inheritance pattern, and early detection of potentially affected relatives. The present study highlights the importance of pedigree analysis in families with FD for identifying other possibly affected relatives and investigating the
\end{abstract}


clinical manifestations. This clinical report included 12 Mexican index cases with confirmed FD diagnosis. We constructed and analyzed their pedigree, and diagnosed FD in 24 affected relatives. Clinical features were similar to those reported for other populations. Pedigree analysis further identified an additional 30 women as possible carriers. We conclude that pedigree construction and analysis is a useful tool to help physicians detect and diagnose relatives at risk for FD, particularly heterozygous females, so that they can receive genetic counseling and early treatment. Mexican families with FD were similar to other populations reported in the literature, and our findings confirmed that heterozygous females can have signs and symptoms ranging from subtle manifestations to the classical severe presentation described in males.

Key words: Fabry disease; Heterozygous; Lysosomal storage disease; Female carrier; Pedigree; X-linked

\section{INTRODUCTION}

Although this is an era of molecular medicine and the Human Genome Project has made available many sophisticated laboratory tests, pedigree analysis remains a powerful, inexpensive, readily available tool to identify individuals at risk for genetic diseases. Pedigree analysis allows earlier detection and management of disease, rational testing, screening for cancer, and fetal assessment in high-risk pregnancies. The construction of a screening pedigree is important to provide comprehensive clinical care, to collect family health information, and to reveal the inheritance pattern (Byers and Ashkenas, 1998), with the objective of preventing a disease or achieving early diagnosis and management of the disease (Brock et al., 2010). Family tree analysis helps to identify disorders that may involve genetic mechanisms such as anticipation, mitochondrial inheritance, and X-linked or dominant homozygous lethality (Bennett et al., 1995).

Fabry disease (FD; MIM 301500) is an X-linked disease of lysosomal storage caused by deficiency of $\alpha$-galactosidase A ( $\alpha$-Gal A; EC 3.2.1.22), an enzyme that functions in globotriaosylceramide $(\mathrm{Gb} 3)$ hydrolysis. FD incidence has been estimated to be 1:40,000 to 1:117,000 male births (Mehta et al., 2004). The prevalence of female carriers of FD mutations has been estimated to be 1:339,000 in the UK (Pinto et al., 2010). The GLA (MIM 300644) gene that encodes the $\alpha$-Gal A enzyme is located on Xq22.1, is $12 \mathrm{~kb}$ in length, and contains seven exons (Pastores and Lien, 2002). To date, over 600 mutations have been identified in the GLA gene, most of which are private, i.e., unique or confined to a family, without "hot spots" (Deegan, 2012). The mutation rate is unknown and no genotype-phenotype correlation has been observed (Schaefer et al., 2005).

FD typically presents in childhood, with an average age of onset of 6-10 years in men and 8.1-14 years in women. On the basis of clinical manifestations, FD can be divided into classical (95\%) and atypical (5\%; cardiac, renal, and neurological) variants. The classical presentation usually begins in childhood or adolescence, reaching the most serious stage of the disease during the third or fourth decade of life. Clinical symptoms include acroparasthesia, angiokeratomas, cornea verticillata, hypohidrosis or anhidrosis, left ventricular hypertrophy (LVH), valvular abnormalities, myocardial ischemia, cerebrovascular accident, diarrhea/constipation, abdominal pain, sudden deafness, tinnitus and kidney failure progressing to end- 
stage renal disease (Whybra et al., 2009). The cardiac variant manifests in the fifth or sixth decade of life with cardiac involvement, including LVH and cardiomegaly in the absence of systemic arterial hypertension, as well as conduction abnormalities; these patients may present with proteinuria but usually do not develop renal failure (Barbey et al., 2006). The renal variant is characterized by late onset proteinuria and progressive renal failure (Schiffmann et al., 2009). The neurological variant presents as isolated stroke or TIA (transient ischemic attack), white matter lesions, and vertebrobasilar dolichoectasia (Brouns et al., 2010).

Traditionally, FD is considered a recessive X-linked condition. Diagnosis and treatment strategies have focused on affected men, while heterozygous females have primarily been considered only as carriers of the mutation, with mild clinical manifestations in some cases (MacDermot et al., 2001a,b). However, accumulated clinical experience with FD has shown the presence of symptoms in women, sometimes as severe as those observed in males. Approximately $60-70 \%$ of heterozygous females have some clinical manifestations (Bennett et al., 2002). To expand our knowledge of FD inheritance and presentation in heterozygous females, we considered it essential to analyze index cases and their families with FD. In the present study, we constructed and analyzed the pedigrees of index patients with FD with the aim of analyzing the clinical manifestations of our patient group and identifying other affected relatives and heterozygous females.

\section{MATERIAL AND METHODS}

This study included 12 index cases referred from the "UMAE Hospital de Especialidades", Centro Médico Nacional de Occidente, Instituto Mexicano del Seguro Social, Guadalajara, México. All patients gave written informed consent to participate in this study, which was performed in adherence with the Declaration of Helsinki, and the Mexican regulations for health and research. This study was approved by the Comisión Nacional de Investigación Científica (R-2011-785-009).

Index cases included 10 men and 2 women. We constructed and analyzed the pedigrees of each proband. Diagnoses of index patients and their affected relatives were based on clinical manifestations and confirmed by biochemical and/or molecular analysis. FD was indicated when $\alpha$-Gal A enzymatic activity in blood was below $6.3 \mathrm{pmol} /(\mathrm{dish} * \mathrm{~h})$ [normal range $6.3-23$ $\left.\mathrm{pmol} /\left(\mathrm{dish}{ }^{*} \mathrm{~h}\right)\right]$. We also performed mutation analysis with an automated sequencing method that screened all seven exons of GLA (ABI PRISM 377 Genetic Analyzer; Applied Biosystems, Foster City, CA, USA). For clinical and statistical analyses, patients were stratified into three groups: men, all heterozygous women (symptomatic and asymptomatic), and symptomatic heterozygous women. Pedigrees and family data were captured with the Cyrillic version 2.1 software. Statistical evaluation was conducted using the SPSS v.20.0 software, and statistical analyses were performed using the $\chi^{2}$ test or Fisher exact test. $\mathrm{P}<0.05$ was considered to be statistically significant.

\section{RESULTS}

This study included 12 index cases (10 males and 2 females), and we further identified 24 affected relatives ( 8 males and 16 females). A total of 36 patients were diagnosed with FD, including $18(50 \%)$ men and $18(50 \%)$ women, of which 7 were asymptomatic and 11 symptom- 
atic. All diagnoses were based on clinical manifestations and then confirmed by enzyme activity test and/or molecular assay in males, and by mutation detection in females; in some instances, women were designated as obligate carriers according to the pedigree. We investigated five generations per family, and the pedigree drawings included a mean of 62.75 individuals per family.

Table 1 displays the demographic features and age of symptom onset in Mexicans with Fabry disease according to gender. We further analyzed the clinical manifestations in patients with FD distributed according to the established groups of males, all females, and only symptomatic females (Table 2). The first clinical manifestation referred by index cases was childhood-onset acroparesthesia, which was also the most common symptom among men and women, reported by 94.4 and 50\%, respectively. The second most common symptom was heat/cold intolerance. Between-group comparisons showed significant statistical differences in angiokeratomas, hypohidrosis/anhidrosis, recurrent fever/impaired temperature regulation, heat/cold intolerance, acroparesthesia, cornea verticillata, abdominal colic pain, proteinuria, and fatigue (Table 3). Some of the patients had previously been misdiagnosed as having rheumatic fever, lupus, vasculitis, fibromyalgia, and hypochondria. Pedigree revisions enabled the identification of 30 additional women who are likely FD heterozygous; these women must be analyzed in further studies to confirm or discard FD diagnosis.

Table 1. Demographic features and age of symptom onset in Mexicans with Fabry disease by gender.

\begin{tabular}{lcc}
\hline Features & \multicolumn{2}{c}{ Means \pm SD (range) } \\
\cline { 2 - 3 } & \multicolumn{1}{c}{ Males } & Females \\
\hline Age (years) & $33.14 \pm 14.17(11-55)$ & $33.71 \pm 18.09(4-63)$ \\
Age of symptom onset (years) & $10.3 \pm 4.15(6-20)$ & $17.63 \pm 10.51(10-36)$ \\
Age of diagnosis (years) & $29.28 \pm 13.25(9-47)$ & $29.38 \pm 17.85(3-60)$ \\
Time delay for diagnosis (years) & $14.53 \pm 11.64(1-37)$ & $10.83 \pm 13.18(1-33)$ \\
\hline
\end{tabular}

Table 2. Clinical presentation in different groups of patients with Fabry disease.

\begin{tabular}{|c|c|c|c|}
\hline Clinical manifestations by system & Male $[\mathrm{N}=18(\%)]$ & $\begin{array}{c}\text { All heterozygous } \\
\text { females }[\mathrm{N}=18(\%)]\end{array}$ & $\begin{array}{c}\text { Symptomatic heterozygous } \\
\text { females }[\mathrm{N}=11(\%)]\end{array}$ \\
\hline \multicolumn{4}{|l|}{ Skin } \\
\hline Angiokeratomata & $7(38.9)$ & $1(5.6)$ & $1(9.1)$ \\
\hline \multicolumn{4}{|l|}{ Nervous system } \\
\hline Hypohidrosis/anhidrosis & $14(77.8)$ & $4(22.2)$ & $4(36.4)$ \\
\hline Impaired temperature regulation & $8(44.4)$ & $2(11.1)$ & $2(18.2)$ \\
\hline Heat/cold intolerance & $15(83.0)$ & $6(33.3)$ & $6(54.5)$ \\
\hline Acroparesthesia & $17(94.4)$ & $9(50.0)$ & $9(81.8)$ \\
\hline \multicolumn{4}{|l|}{ Ophthalmological } \\
\hline Cornea verticillata & $8(44.4)$ & $3(16.7)$ & $3(27.3)$ \\
\hline \multicolumn{4}{|l|}{ Gastroenterological } \\
\hline Diarrhea/constipation & $9(50.0)$ & $7(38.9)$ & $7(63.6)$ \\
\hline Abdominal colic pain & $10(55.6)$ & $4(22.2)$ & $4(36.4)$ \\
\hline \multicolumn{4}{|l|}{ Renal } \\
\hline Proteinuria & $13(72.2)$ & $5(27.8)$ & $5(45.5)$ \\
\hline Progressive renal impairment & $6(33.3)$ & $2(11.1)$ & $2(18.2)$ \\
\hline \multicolumn{4}{|l|}{ Cerebrovascular } \\
\hline Stroke & $3(16.7)$ & - & - \\
\hline \multicolumn{4}{|l|}{ Cardiovascular } \\
\hline Left ventricular hypertrophy & $3(16.7)$ & $2(11.1)$ & $2(18.2)$ \\
\hline \multicolumn{4}{|l|}{ Auditory } \\
\hline Sudden deafness & $7(38.9)$ & $5(27.8)$ & $5(45.5)$ \\
\hline Tinnitus & $7(38.9)$ & $5(27.8)$ & $5(45.5)$ \\
\hline \multicolumn{4}{|l|}{ Miscellaneous } \\
\hline Fatigue & $13(72.2)$ & $3(16.7)$ & $3(27.2)$ \\
\hline
\end{tabular}




\begin{tabular}{lcc}
\multicolumn{2}{c}{ Table 3. Comparative analysis by groups in Fabry disease. } \\
\hline Clinical manifestation & Group 1 vs Group 2 P value & Group 1 vs Group 3 P value \\
\hline Angiokeratomata & $\mathbf{0 . 0 4 1 *}$ & 0.110 \\
Hypohidrosis/anhidrosis & $\mathbf{0 . 0 0 2}^{*}$ & $\mathbf{0 . 0 4 8}$ \\
Impaired temperature regulation & $\mathbf{0 . 0 2 3} *$ & 0.124 \\
Heat/cold intolerance & $\mathbf{0 . 0 0 6 *}$ & 0.197 \\
Acroparesthesia & $\mathbf{0 . 0 0 7 *}$ & 0.539 \\
Cornea verticillata & $\mathbf{0 . 0 2 7} *$ & 0.246 \\
Diarrhea/constipation & 0.505 & 0.705 \\
Abdominal pain & $\mathbf{0 . 0 4 1 *}$ & 0.440 \\
Proteinuria & $\mathbf{0 . 0 1 8 *}$ & 0.240 \\
Progressive renal impairment & 0.228 & 0.435 \\
Left ventricular hypertrophy & 0.656 & 1.0 \\
Deafness & 0.300 & 1.0 \\
Tinnitus & 0.475 & 1.0 \\
Fatigue & $\mathbf{0 . 0 0 2 *}$ & 0.060
\end{tabular}

Group 1 = males; Group 2 = all heterozygous female; Group 3 = symptomatic heterozygous female. $*$ Statically significant.

\section{DISCUSSION}

Fabry disease, an X-linked lysosomal storage disorder, has been traditionally seen as mainly affecting men, with women being only mutation carriers; however, recent studies have shown that carriers can be symptomatic (Barba-Romero et al., 2010). In such instances, diagnosis of the index case is often delayed and carrier diagnosis is often undervalued. A properly used pedigree can be a great tool, enabling the clinician to diagnose affected relatives in a timely manner, thus allowing early onset of enzyme replacement therapy, which is the basis of a favorable prognosis.

In the present study, two of the index patients were women, confirming that women and men can be equally affected and that men can be diagnosed based on the diagnosis of a heterozygous female. Among all patients, the three most frequent and consistent clinical features were acroparesthesia, heat/cold intolerance, and hypohidrosis/anhidrosis. No significant differences were found between men and symptomatic heterozygous females - only a marginal significance for hypohidrosis frequency $(\mathrm{P}=0.048)$. Thus, a symptomatic heterozygous female manifests symptoms much like a man with Fabry disease. However, when comparing men with all heterozygous women (symptomatic and asymptomatic), we found statistically significant differences in angiokeratoma, hypohidrosis, impaired temperature regulation, heat/ cold intolerance, acroparesthesia, verticillata cornea, abdominal colic pain, and proteinuria. Of all 18 carriers, $61.1 \%$ showed at least one symptom and only 7 were asymptomatic, demonstrating the importance of monitoring and diagnosing carriers. Proteinuria was observed in both groups (in $72.2 \%$ of men and $27.8 \%$ of women), reflecting the importance of periodically measuring renal function to prevent progression to chronic renal failure, which is a major complication and cause of death in patients with FD. In Table 4, we compare the presently observed frequencies with other reports in the literature.

It is important to highlight the substantial delay between the onset of symptoms and diagnosis: $14.53 \pm 11.64$ years in males and $10.83 \pm 13.18$ years in females. Our patients visited an average of 12 physicians before receiving a definitive diagnosis, which generates anxiety, worry, and stress in the patient and family. We also found that a total of 20 spontaneous abortions occurred in the 12 families studied, with the highest number being 11 abortions in one family; this finding should be further investigated. 


\begin{tabular}{|c|c|c|c|c|c|c|}
\hline Clinical manifestation & Male $^{\mathrm{a}}$ & Male $^{b}$ & $\mathrm{P}$ value & Heterozygous female ${ }^{a}$ & Heterozygous female $^{c}$ & $P$ value \\
\hline Angiokeratomata & $7 / 18$ & $52 / 73$ & 0.010 & $1 / 18$ & $21 / 60$ & $0.032 *$ \\
\hline Hypohidrosis/anhidrosis & $14 / 18$ & $29 / 52$ & 0.062 & $4 / 18$ & $20 / 60$ & $0.405^{*}$ \\
\hline Acroparesthesia & $17 / 18$ & $72 / 93$ & $0.117 *$ & $9 / 18$ & $42 / 60$ & 0.117 \\
\hline GI symptoms & $10 / 18$ & $48 / 70$ & 0.298 & $7 / 18$ & $35 / 60$ & 0.146 \\
\hline Proteinuria & $13 / 18$ & $37 / 44$ & 0.282 & $5 / 18$ & - & - \\
\hline Progressive renal failure & $6 / 18$ & $28 / 60$ & 0.317 & $2 / 18$ & $7 / 20$ & $0.130 *$ \\
\hline TIA or CVA & $3 / 18$ & $17 / 70$ & $0.552 *$ & $0 / 18$ & $13 / 60$ & $0.064 *$ \\
\hline LVH & $3 / 18$ & $30 / 34$ & 0.040 & $2 / 18$ & $4 / 21$ & $0.667 *$ \\
\hline Sudden deafness & $7 / 18$ & $25 / 61$ & 0.873 & $5 / 18$ & $14 / 60$ & 0.700 \\
\hline Tinnitus & $7 / 18$ & $23 / 60$ & 0.602 & $5 / 18$ & $15 / 60$ & 0.812 \\
\hline Fatigue & $13 / 18$ & $40 / 70$ & 0.243 & $3 / 18$ & $40 / 60$ & $<0.001 *$ \\
\hline
\end{tabular}

GI = gastrointestinal; TIA $=$ transitory ischemic attack; CVA $=$ cerebrovascular accident; LVH $=$ left ventricular hypertrophy; *Fisher's exact test; ${ }^{a}$ Our data; ${ }^{b}$ Macdermot et al., 2001a; ${ }^{~}$ Macdermot et al., 2001b. P value data in bold are statiscally significant.

One limitation of the present study was the small number of index patients. Due to the low incidence of FD and the frequency of initial misdiagnosis, there are a limited number of known affected individuals. Longer periods of time are required to recruit enough probands for a larger study.

The information presented in this study should be of interest to a broad readership, including medical personnel who are interested in improving their knowledge of Fabry disease in both genders, and the identification of such patients using pedigree analysis. Developing an appropriate diagnostic for Fabry disease requires studies of clinical records and of an inherited trait in a group of related individuals to determine the pattern and characteristics of the disease, together with molecular and biological markers. We strongly recommend that physicians carefully observe the female members of these families.

\section{CONCLUSIONS}

In the present study, pedigree analyses allowed us to identify 30 possible carriers who remain to be assessed to confirm or rule out a diagnosis of FD. We also found wide inter- and intra-patient variability of clinical manifestations. Results of the present analysis of clinical manifestations are consistent with previous reports and indicate that inheritance of FD within Mexican families occurs as reported in the literature.

There remains a wide gap between the onset of manifestations and the diagnosis of FD. Early diagnosis improves the ability to provide appropriate genetic counseling, and to prevent or treat complications with timely interventions and monitoring. There are established benefits of early intervention with enzyme replacement therapy in patients with FD, including reversal of cellular damage, and significant improvements in symptoms and quality of life. Thus, we consider it necessary to investigate the family tree to evaluate and provide early diagnosis for family members at risk.

\section{ACKNOWLEDGMENTS}

Research supported by the National Council of Science and Technology (CONACYT) and Instituto Mexicano del Seguro Social (IMSS) through a Ph.D. scholarship awarded to B. E. Gutiérrez-Amavizca. 


\section{REFERENCES}

Barba-Romero MA, Deegan P, Giugliani R and Hughes D (2010). Does geographical location influence the phenotype of Fabry disease in women in Europe? Clin. Genet. 77: 131-140.

Barbey F, Brakch N, Linhart A, Rosenblatt-Velin N, et al. (2006). Cardiac and vascular hypertrophy in Fabry disease: evidence for a new mechanism independent of blood pressure and glycosphingolipid deposition. Arterioscler. Thromb. Vasc. Biol. 26: 839-844.

Bennett RL, Steinhaus KA, Uhrich SB, O’Sullivan CK, et al. (1995). Recommendations for standardized human pedigree nomenclature. Pedigree Standardization Task Force of the National Society of Genetic Counselors. Am. J. Hum. Genet. 56: 745-752.

Bennett RL, Hart KA, O’Rourke E, Barranger JA, et al. (2002). Fabry disease in genetic counseling practice: recommendations of the National Society of Genetic Counselors. J. Genet. Couns. 11: 121-146.

Brock JA, Allen VM, Keiser K and Langlois S (2010). Family history screening: use of the three generation pedigree in clinical practice. J. Obstet. Gynaecol. Can. 32: 663-672.

Brouns R, Thijs V, Eyskens F, Van den Broeck M, et al. (2010). Belgian Fabry study: prevalence of Fabry disease in a cohort of 1000 young patients with cerebrovascular disease. Stroke 41: 863-868.

Byers PH and Ashkenas J (1998). Pedigrees-publish? or perish the thought? Am. J. Hum. Genet. 63: 678-681.

Deegan PB (2012). Fabry disease, enzyme replacement therapy and the significance of antibody responses. J. Inherit. Metab. Dis. 35: 227-243.

MacDermot KD, Holmes A and Miners AH (2001a). Anderson-Fabry disease: clinical manifestations and impact of disease in a cohort of 98 hemizygous males. J. Med. Genet. 38: 750-760.

MacDermot KD, Holmes A and Miners AH (2001b). Anderson-Fabry disease: clinical manifestations and impact of disease in a cohort of 60 obligate carrier females. J. Med. Genet. 38: 769-775.

Mehta A, Ricci R, Widmer U, Dehout F, et al. (2004). Fabry disease defined: baseline clinical manifestations of 366 patients in the Fabry Outcome Survey. Eur. J. Clin. Invest. 34: 236-242.

Pastores GM and Lien YH (2002). Biochemical and molecular genetic basis of Fabry disease. J. Am. Soc. Nephrol. 13 (Suppl 2): S130-S133.

Pinto LL, Vieira TA, Giugliani R and Schwartz IV (2010). Expression of the disease on female carriers of X-linked lysosomal disorders: a brief review. Orphanet. J. Rare Dis. 5: 14.

Schaefer E, Mehta A and Gal A (2005). Genotype and phenotype in Fabry disease: analysis of the Fabry Outcome Survey. Acta Paediatr. Suppl. 94: 87-92.

Schiffmann R, Warnock DG, Banikazemi M, Bultas J, et al. (2009). Fabry disease: progression of nephropathy, and prevalence of cardiac and cerebrovascular events before enzyme replacement therapy. Nephrol. Dial. Transplant. 24: 2102-2111.

Whybra C, Miebach E, Mengel E, Gal A, et al. (2009). A 4-year study of the efficacy and tolerability of enzyme replacement therapy with agalsidase alfa in 36 women with Fabry disease. Genet. Med. 11: 441-449. 